Journal of Education and Vocational Research

Vol. 2, No. 2, pp. 34-37, Aug 2011 (ISSN 2221-2590)

\title{
Review of Harvard Business Review on Collaborating Across Silos
}

\section{Book Review}

\author{
Reviewed by: Shiva Kumar Srinivasan \\ Institute of Planning and Management (IIPM), Chennai \\ sksrinivasan2008@hotmail.com
}

The need to shift the fundamental orientation from a product focus to a customer focus in contemporary firms means that the different functions and divisions therein must learn to operate with the ethic of 'boundarylessness'. This is the re-organizational ethic that Jack Welch pushed relentlessly during his time at GE. The academic equivalent of this notion in management theory is known as 'collaborating across silos'. Each organizational silo then can be understood as a function, division, or vertical depending on the type of firm that we are describing. The shift in focus from products to customers and services means that is important for firms to understand that organizational re-design is not an end itself but rather an organizational means to a strategic end. But, even given the strategic importance of collaborating across silos, this remains easier said than done. This is because the employee's sense of professional identity is related to an area of specialization which is not easy to dislodge either because the employee has stopped learning, or does not have sufficient exposure to the remaining areas, or has not been given any opportunity to partake of job enrichment, job rotation, or cross-training that is presupposed in the attempt to build-in the competencies required to collaborate across silos. The usual situation in firms then is that the need for effective collaboration does not get translated into actual customer-based interventions that add value on a sustainable basis. Instead, there are sporadic attempts at collaboration that will sooner or later be reduced or nullified altogether because of the insecurities and inadequacies in the repertoire of skill-sets that are available in a given firm. This then is the challenge that the contributors to this anthology of essays from the Harvard Business Review have taken up. Most of the contributors are either business academics or management consultants who are increasingly worried about this problem and the need to take remedial measures to correct it before customer dissatisfaction forces the issue onto the strategic agenda of firms.

Lynda Gratton of the London Business School and Tamara J. Erickson of genera draw up eight important approaches to building teams that can collaborate across silos. They recognize that the main difficulty in coordinating such teams comes not only from the routine problem of co-ordination costs, but also from the ever increasing costs of complexity. Their advice here is based on a research survey in which 1,543 respondents participated across domains, functions, and 55 firms. The main goal was to identify how much leeway executives in these firms had to set up and make a success of collaborative teams of which the average size was 44. Amongst the possible organizational levers that were examined to determine the extent of strategic and operational leeway included organizational culture, HR practices, modes of employee socialization, task design, and the forms of leadership in the organization. The main challenges that were identified include the increasing size of firms, socio-cultural diversity of team members, the levels of training and education, and the recent emergence of virtual teams. The eight approaches that they advocate in the aftermath of this study comprise the need to develop 'signature relationship practices' (which demonstrate the symbolic commitment of the firm's leadership to collaborative activities), attempts to 'model collaborative behavior' by senior members of the hierarchy, the cultivation of a 'gift culture' (i.e. one where 'coaching and mentoring' by seniors is attempted without expecting much in return), training employees in the skills needed to excel by HR professionals, creating a strong 'sense of community' to facilitate knowledgesharing, encouraging team leaders to work with both task skills and relationship skills, incentivizing team members who already know each other to collaborate in the first instance, and ensuring that there is sufficient clarity on role expectations. This may seem like an impossible order but Philip Evans and Bob Wolf of the Boston Consulting Group point out instances of effective self-organizing communities such as those who work free of cost to develop code for Linux, and those who work for a consideration in the context of the Toyota Production System. These are forms of team-based empowerment that most people will find hard to believe especially in their ability to respond to emergencies (for instance they may have to produce security 
patches for software or stop the assembly line to address quality issues). Understanding the motivational structure of such self-organizing teams and understanding what, if anything prompts them to sustain such levels of employee initiative and engagement, will provide useful lessons for those academics and consultants working in this area. They therefore work out the characteristic features of these teams on the assumption that this will lead to effective emulation of such practices by the readers of this book. What these teams have in common are not only high levels of trust, but access to collaborative technology, an understanding of granularity, and the ability to think in terms of modularity. While this is not a complete list, it captures at least the most important features of such teams.

Jeff Weiss and Jonathan Hughes of Vantage partners add a note of caution to these discussions on building effective teams; they point out that effective collaboration is not something that happens in the absence of conflict (since intelligent people will disagree), but on the ability to manage and channelize these conflicts effectively. A creative conflict that is managed effectively will build genuine rapport and make it possible for a team to collaborate both within on-line and off-line communities whereas a conflict that is not recognized to exist at all will be displaced onto innumerable little problems evading the onset of the camaraderie that is being sought to manage customers and clients through cross-functional collaboration. Michael E. Raynor of Deloitte Consulting and Joseph L. Bower of Harvard Business School point out that while a number of techniques have been tried to build collaborative teams, it has not been possible to effectively do away with silo mind-sets. Since large firms are built as a collection of divisions, it is important in turbulent markets for headquarters to be actively involved in managing them. They invoke four important case studies to argue that a firm can be much more dynamic in its ability to generate synergies across divisions if it is flexible in terms of how the divisions are organized. The organizational design that is used nowadays is to either completely relate or separate the divisions. It might however be a better idea to have a varying set of organizational 'relatedness' between the divisions in a firm; they refer to these organizational mechanisms as 'strategic flexibility'. These problems of divisional organization affect diversified companies even more. It is therefore important to move from 'static to dynamic' forms of thinking so that what is defined as 'corporate value' is not reducible to what happens in business units but also happens at the strategic level. In the dynamic approach to interdivisional relations, the 'degree of relatedness waxes and wanes depending upon strategic circumstances' making it thereby possible to attend to the strategic needs of the firm as a whole. This is all the more important since collaboration across silos is not an organizational ideal per se, but because there is a fundamental difference between operating in 'stable and rapidly changing environments'. The strategic approaches of the firm must therefore reflect the need to address the 'institutional voids', if any, (as Tarun Khanna of HBS and institutional economists might put it), in the business environment.

What is at stake then is customer focus rather than organizational design as a source of professional identity for employees. Ranjay Gulati of HBS points out that re-organizing the product portfolio to include services is easier said than done. There is no easy formulation to make this transition even in companies that have a customer focus; it is therefore important to offer consultancy services as an additional feature in a way that is not reducible to the form of a 'product accompaniment' in the attempt to minimize commoditization of the product range. This is the strategy that GE Healthcare used, for instance, to make its 'performance solutions' more viable; this helps to prevent a firm from being too preoccupied with its own product line and more likely to incorporate features from elsewhere in a given domain. In order to attain the levels of customer focus that Gulati is asking for, firms will have to learn to 'co-ordinate and co-operate' across teams; they will have to develop the capabilities required in terms of skill-sets; and above all, they will have to build 'relationships with external partners to increase the value of solutions cost effectively'. The kind of skill-sets that is at stake here was first identified in the context of knowledge management in a case on McKinsey \& Company by Christopher Bartlett of HBS. A 'T-shaped' consultant, in the context of McKinsey, was one who had both deep vertical expertise and the ability to understand its applications across verticals (or practice lines). Here the term is used mainly in terms of knowledge-sharing 'behavior' that enhances collaboration across silos rather than in terms of an executive's 'skill-base' per se. Morton T. Hansen of INSEAD and Bolko Von Oetinger of the Boston Consulting Group argue that such T-shaped executives will make it possible to share explicit forms of knowledge though it is not clear how 'tacit' forms of knowledge must be shared. That then is the next challenge in thinking through the challenges of collaboration since tacit knowledge evades the strategies of knowledge management that are currently available to capture what a firm and its employees know. The difficulties of doing this are discussed in the context of BP's merger with Amoco and ARCO. Here 
the problem was that knowledge sharing became an end in itself rather than as a means to addressing specific instances of customer management. While this is bound to be the case when a new practice is initiated, it is important to get a grip on what all this will cost the firm. In BP, employees were given credit for helping out elsewhere under the aegis of team assist/peer assist, which would attempt to calculate the addition of 'horizontal value' over and above the vertical to which the employee was originally assigned. Collaborative forms of value addition were situated on the value chain in terms of both 'traditional knowledge transfer', and in terms of creating 'new ideas'. The gains included increase in levels of effectiveness, quality of decision making, growth in revenues, developing new businesses, and better implementation of strategic initiatives. Employees who would mediate the quest for ideas, knowledge, skill-sets, and could then match questions from a division with answers from another division became 'human portals at work'. A good instance of this is produced in the essay as a series of email exchanges in which Larry Watson and Les Owen try to find a solution for protecting pipelines from 'lightning strikes' by contacting those engineers in the know elsewhere in the firm. Hansen and Oetinger also discuss how T-shaped approaches to management are being used in firms like GlaxoSmithKline, Siemens, and Ispat International.

While these case studies and examples are persuasive, there is no guarantee that they will work elsewhere unless we can identify the 'tools of cooperation and change'. Knowing 'what' tool to use 'when' is what is at stake in deploying these tools effectively. Clayton M. Christensen of HBS et al argue that it is important to see if there is agreement on what is wanted and how this want is approached. He invokes an agreement matrix in which any decision making opportunity can be situated. Whether tools of cooperation or power are invoked then depends on how a problem is situated in the agreement matrix. In this matrix, the ' $x$ axis' pertains to the degree of consensus on how cause and effect is understood in a firm; the 'y axis' relates to the extent of consensus on the strategic agenda. The four possible tools of cooperation which include 'power, management, leadership, and culture' are deployed based on how a problem is situated in the agreement matrix. Power tools are more likely to be used when there is disagreement on what must be done; leadership tools are more likely to be used when it is not clear how something must be executed. Jack Welch used power tools when he admonished his managers to be in the top two in a given domain failing which they would be asked to close shop. Welch's success in reorganizing GE makes it much more likely that it will suffice if Jeff Immelt were to use only leadership tools. Analogously, the fact that Gordon Bethune could reorganize Continental Airways using power tools makes it much more likely that Larry Kellner, his successor, will need to deploy only leadership tools to elicit the cooperation of his employees. Important instances of how communication techniques can be harnessed as leadership tools include the well-known 'Internet Tidal Wave Memo of 1995' and the 'Services Wave Memo of 2005' that Bill Gates used at Microsoft to make his case for the importance of the Net, and in emphasizing the need to move from a product orientation to a services orientation respectively. And, finally, we presuppose that the willingness to be amenable to such tools requires not only a high level of emotional intelligence in the leadership but also amongst employees, team leaders, and teams.

While most discussions on matters pertaining to emotional intelligence are specific to individuals, this is increasingly being recognized as a prerequisite to effective team work as well since it requires the subordination of the ego in the interests of the greater good. Being able to handle the ego without taking things in too emotional a way are often cited as manifestations of emotional intelligence. Hence, Vanessa Urch Drusskat of the Whitmore School of Business and Steven B. Wolff of Haygroup Consultants argue that it is imperative to build emotional intelligence in groups with as much urgency as in individual employees. They invoke the self-regulation of affects by both individuals and groups as a test case for the level of emotional intelligence at work in any given situation. Emotional intelligence is understood as both 'personal competence' and as 'social competence'. The former pertains to how individuals perceive and regulate their inner world; the latter relates to how the affects exhibited by others can be effectively regulated to prevent escalation of misunderstandings and maintain focus on the key agenda items in social interactions. The value of these skills should be obvious both for team leaders and team members: unless both forms of competence are present, it will not be possible for the team to move forward. While these forms of competence can cover for each other every now and then, sustained progress is only possible if both are present and the team members and leaders are willing to learn from each other. Group-based affects/emotions can be contagious; it is all the more important for a team to be actively aware of these so that they can be effectively regulated to minimize any kind of fall-out in trying situations. There are three levels at which the manifestations of emotional intelligence can be studied. They are 'trust, identity, and efficacy' at the first level; 'participation, 
co-operation, and collaboration' at the second level; and, finally, 'better decisions, more creative solutions, and higher productivity' at the third level. A team can be described as emotionally intelligent only if it is able to appreciate, understand, and operate at all the three levels described in this framework. These levels also constitute, as it were, the organizational preconditions for 'collaborating across silos' in contemporary firms. 